\title{
Modeling of Reactive Diffusion: Mechanism and Kinetics of the Intermetallics Growth in $\mathrm{Ag} / \mathrm{Ag}$ Interconnections
}

\author{
R. Filipek, K. Szyszkiewicz, P. Dziembaj, P. Skrzyniarz, A. Wierzbicka-Miernik, and P. Zieba
}

(Submitted November 24, 2011; in revised form December 22, 2011)

\begin{abstract}
The phenomenological model describing the growth of intermetallic phases in multi-component systems is presented. Full time-dynamics approach is applied without the often-used simplifications such as flux constancy. General form of the species flux is considered, which consists of chemical potential gradient as a driving force for diffusion with additional drift term. Stefan-type (moving) boundary conditions are applied. In the present form, the model assumes local equilibrium at each interface and that the process of growth of intermediate phases is controlled by diffusion of reagents through the layers and/or chemical reactions at the boundaries. The model is solved in its full generality. Numerical method for the solution of the problem has been developed. Specially selected change of dependent variables transforms the moving boundary problem into an equivalent fixed boundary problem. Such problem has been treated using the method of lines which converts partial differential equations into a system of ordinary differential equations, which is subsequently solved numerically. The obtained solution was tested and compared with analytic ones available in special cases, showing satisfactory agreement. The growth of intermetallic phases in $\mathrm{Ag} / \mathrm{Sn} / \mathrm{Ag}$ system has been modeled and compared with experimental results.
\end{abstract}

Keywords intermetallics, joining, modeling processes

\section{Introduction}

Diffusion soldering technology is an effective method of obtaining stable metal/metal interconnections using solder which forms intermetallic phase with the joining materials. The joint microstructure, chemical composition, and the sequence of appearance of the intermetallic phases are important factors that influence its stability. The important parameters determining diffusion soldering technology are the thickness of the solder, duration, and temperature of the process. Understanding of the mechanism of this process is necessary for

This article is an invited submission to JMEP selected from presentations at the Symposia "Wetting, soldering and brazing" and "Diffusion bonding and characterization" belonging to the Topic "Joining" at the European Congress and Exhibition on Advanced Materials and Processes (EUROMAT 2011), held September 12-15, 2011, in Montpellier, France, and has been expanded from the original presentation.

R. Filipek, K. Szyszkiewicz, and P. Dziembaj, Faculty of Materials Science and Ceramics, Interdisciplinary Centre of Materials Modeling, AGH University of Science and Technology, 30 Mickiewicza Av., 30-059 Kraków, Poland; K. Szyszkiewicz, Faculty of Computer Sciences, WSB-NLU, Zielona 27, 33-300 Nowy Sącz, Poland; and P. Skrzyniarz, A. Wierzbicka-Miernik, and P. Zięba, Institute of Metallurgy and Materials Science, Polish Academy of Sciences, 25 Reymonta St., Kraków, Poland. Contact e-mail: rof@agh.edu.pl. prediction of growth kinetics of phases and, finally, optimization of the diffusion soldering technology.

In this article mathematical model of the process is formulated. Method of solving moving-boundary problem is developed. Numerical solution of the problem is verified against analytic solutions. Comparison of the model with experimental results is presented.

\section{Mathematical Model}

A model of reactive diffusion describing the growth of intermediate phases in multi-component systems in one-dimensional geometry is presented below. It includes data, physical laws of transport processes, initial conditions, boundary conditions, and unknowns.

\subsection{Data}

1. Diffusion coefficients of components $(i=1, \ldots, r)$ in different phases $(\alpha=1, \ldots, f)$ as functions of the molar fraction: $D_{i}^{\alpha}\left(N_{1}, \ldots, N_{r}\right)$, where $r$ and $f$ denote the number of components (species) and phases, respectively.

2. Initial positions of the phase boundaries: $s_{0}(0), \ldots$, $s_{\alpha}(0), \ldots, s_{f}(0)$.

3. Activities of the components in all phases as functions of the molar fractions of components: $a_{i}^{\alpha}\left(N_{1}, \ldots, N_{\mathrm{r}}\right)$, where $i=1, \ldots, r ; \alpha=1, \ldots, f$.

4. Duration (time) of the process: $\hat{t}$.

\subsection{Physical Laws}

Mass transport in every phase is governed by the local mass balance (continuity equation) (Ref 1$)$ : 


$$
\begin{gathered}
\frac{\partial c_{i}^{\alpha}}{\partial t}(x, t)=-\frac{\partial J_{i}^{\alpha}}{\partial x}(x, t) \quad s_{\alpha-1}(t)<x<s_{\alpha}(t), \quad t \in[0, \hat{t}] \\
i=1, \ldots, r, \quad \alpha=1, \ldots f .
\end{gathered}
$$

where $c_{i}^{\alpha}$ and $J_{i}^{\alpha}$ denote concentration $\left(\mathrm{mole} / \mathrm{m}^{3}\right)$ and flux (mole $/ \mathrm{m}^{2} \mathrm{~s}$ ) of the $i$ th component in the phase $\alpha$, respectively.

The flux $J_{i}^{\alpha}$ in Eq 1 will be expressed as a sum of diffusive flux $\left(J_{i}^{\alpha, d}\right)$ and drift flux $\left(c_{i} \mathrm{v}\right)$. Hence,

$J_{i}^{\alpha}=J_{i}^{\alpha, d}+c_{i}^{\alpha} v^{\alpha}$.

The diffusive part of the flux is given by the Nernst-Planck expression (Ref 2, 3)

$J_{i}^{\alpha, d}=B_{i}^{\alpha} c_{i}^{\alpha} \sum_{j} F_{j}$,

where $B_{i}^{\alpha}$ is the mobility of the $i$ th component in the phase $\alpha$, and $\sum_{j} F_{j}$ is the sum of thermodynamic forces causing diffusion. In the isothermal-isobaric conditions a diffusion flow is generated by the gradient of chemical potential, $\mu_{i}^{\alpha}$ (Ref 4). Thus, we have the following form of the diffusion flux:

$J_{i}^{\alpha, d}=-B_{i}^{\alpha} c_{i}^{\alpha} \frac{\partial \mu_{i}^{\alpha}}{\partial x}$

In general, the chemical potential may be dependent on all species in the system. By using the chain rule for derivatives, we can express (4) as sum of gradients of concentrations (or molar fractions) of components (Ref 5)

$J_{i}^{\alpha, d}=-\sum_{j=1}^{r} D_{i j}^{\alpha} \frac{\partial c_{j}^{\alpha}}{\partial x}$,

where partial intrinsic diffusion coefficients $D_{i j}^{\alpha}$ in phases $\alpha=1, \ldots, f$ are defined by the formulas:

$D_{i j}^{\alpha}:=D_{i}^{\alpha} c_{i}^{\alpha} \frac{\partial \ln a_{i}^{\alpha}}{\partial c_{j}^{\alpha}}$.

In some circumstances, for instance, when the growing phase is still very thin, the gradient can be very large, and as a consequence, the classical (continuous) description may lead to the paradox of "infinite" flux. On the other hand, the microscopic "picture" of diffusion is based on the jumps of atoms (Ref 6). The length of the jump determines the maximum feasible diffusion flux $J_{i \text {, max }}^{d}(\operatorname{Ref} 7)$ :

$J_{i, \max }^{d}=D_{i} c_{i} / \lambda_{i}$

where $\lambda_{i}$ denotes the characteristic distance for the diffusion (jump distance of a defect). The phase exists if and only if the diffusion flux in this phase does not exceed $J_{i \text {, max }}^{d}$. Thus, this condition can be written as

$J_{i}^{d} \leq J_{i, \max }^{d}=D_{i} c_{i} / \lambda_{i}$,

The constraints on the maximum value of the flux were earlier applied in the hydromechanics problems (Ref 8, 9), plasma physics modeling (Ref 10), and for charge transport processes in microelectronic systems (Ref 11, 12). The kinetic constraint (8) for inter-diffusion problems of multicomponent systems was applied also by Danielewski and Wakihara (Ref 7).

Thus, in this model, we will make use of the constraints on the diffusion fluxes in each phase:
$J_{i}^{\alpha, d} \leq J_{i, \max }^{\alpha, d}=D_{i}^{\alpha} c_{i}^{\alpha} / \lambda_{i}^{\alpha} \quad i=1, \ldots, r, \quad \alpha=1, \ldots, f$

\subsection{Initial and Boundary Conditions}

2.3.1 Initial Conditions. The model permits the use of any initial concentration $c_{i}^{\alpha}(0, x)=c_{i}^{\alpha, 0}(x)$ in each phase $\alpha$. In diffusion soldering, the initial compositions are usually simple, for example, constant concentrations in the substrate and in the solder, $c_{i}^{\alpha, 0}$ and $c_{i}^{\gamma, 0}$, respectively.

2.3.2 Boundary Conditions. The flux balance equations at the interfaces can be formulated as follows:

$$
\begin{gathered}
\left(c_{i}^{\alpha}\left(s_{\alpha}(t), t\right)-c_{i}^{\alpha-1}\left(s_{\alpha}(t), t\right)\right) \frac{d s_{\alpha}}{d t} \\
=J_{i}^{\alpha}\left(s_{\alpha}(t), t\right)-J_{i}^{\alpha-1}\left(s_{\alpha}(t), t\right), \\
i=1, \ldots, r, \quad \alpha=1, \ldots, f-1,
\end{gathered}
$$

where $s_{\alpha}(t)$ is the moving position of the phase boundary $(\alpha-1 \mid \alpha)$ at time $t ; c_{i}^{j}\left(s_{k}(t), t\right)$, and $J_{i}^{j}\left(s_{k}(t), t\right)$ are the concentration and flux, respectively, of the $i$ th species in $j$ th phase at the $k$ th boundary. The boundary conditions may be imposed for each species at each phase boundary.

If reactions at the interface are fast-compared to the velocities of the phase interfaces - then local thermodynamic equilibrium can be assumed to hold at the interfaces. Consequently, we can demand that chemical potentials of components in both phases must be the same:

$$
\begin{gathered}
\mu_{i}^{\alpha-1}\left(c_{1}^{\alpha-1}, \ldots, c_{r}^{\alpha-1}\right)=\mu_{i}^{\alpha}\left(c_{1}^{\alpha}, \ldots, c_{r}^{\alpha}\right), \\
i=1, \ldots, r \quad \text { and } \quad \alpha=1, \ldots, f .
\end{gathered}
$$

By using $\mathrm{Eq} \mathrm{11,} \mathrm{the} \mathrm{equilibrium} \mathrm{concentrations} \mathrm{at} \mathrm{the}$ interfaces for the boundary conditions (10) can be determined. Subsequently, the diffusion problem given by Eq 1-11 can be solved for each single-phase region. The velocities and positions of the interfaces are determined by solving the fluxbalance equations (10).

\subsection{Unknowns}

1. Positions of phase boundaries as functions of time: $s_{0}(t), \ldots, s_{f}(t)$ for $t \in[0, \hat{t}]$.

2. Concentrations profiles $c_{i}^{\alpha}(x, t), i=1, \ldots, r$ in each phase $\alpha=1, \ldots, f$ for $x \in\left[s_{\alpha-1}(t), s_{\alpha}(t)\right]$ and $t \in[0, \hat{t}]$.

2.4.1 Solution of the Model. Equations describing the growth of multi-component layers form a system of nonlinear partial differential equations. Moreover, situation is complicated further because the positions of interfaces change with time, giving rise to the Stefan-like problems. Hence, we will look for a suitable numerical solution using the finite difference method.

\subsection{Numerical Solution}

Theoretical treatment of growth of phases in diffusion soldering process involves the solution of the so-called Stefan problem (Ref 13). The growth rate is calculated from a mass balance equation at the moving interface and suitable mass balance equations in each phase. The problem is complicated by the fact that the position of phase interface is also an unknown. 
With the exception of simple cases — where analytic solutions are known (Ref 13) — numerical methods must be applied. Extensive and detailed overview of these methods is provided, for example, in Ref 14. In connection with our development, we mention here only the Murray-Landis and enthalpy methods.

The first method was proposed by Murray and Landis (Ref 15). It is a finite difference method which uses contracting and expanding grids. A time-dependent grid allows the phase interface to be always located on a grid point. The number of grid points is constant. The motion of grid points is taken into account by applying the following formula:

$$
\frac{c_{k-1}-2 c_{k}+c_{k+1}}{h^{2}}+s^{\prime} \frac{n-k}{n} \frac{c_{k+1}-c_{k-1}}{2 h}, \quad k=0, \ldots, n,
$$

for discretization of the space second derivative $\partial^{2} c / \partial x^{2}$. The velocity $s^{\prime}$ is obtained from the flux balance at the interface, $\left(c^{\alpha+1}-c^{\alpha}\right) s^{\prime}=J^{\alpha+1}-J^{\alpha}$ using one-sided finite differences, for example,

$$
J^{\alpha+1}=-D^{\alpha+1} \frac{-3 c_{0}+4 c_{1}-c_{2}}{2 h} .
$$

The enthalpy method uses a fixed grid. At each time step, we keep information about the grid points between which the interface is located. Other points belong to different adjacent phases. After advancing in time the concentration profile, a set of rules is applied to assign anew the grid points to one or second phase, or to the interface region. Because at each step we know only two adjacent points between which the interface is located, some interpolation formula is used to obtain the approximate position of the interface. For example, if $i$ is the grid point closest to the interface, the position may be calculated as

$$
s=\left(i+0.5-\frac{c_{i}-c^{\alpha}}{c^{\alpha+1}-c^{\alpha}}\right) h,
$$

where $h$ is distance between grid points, $c^{\alpha+1}$ and $c^{\alpha}$ are the concentrations close to the interface.

The aim of our method was simplicity of presentation. From what has just been described, we can see that above methods are relatively obscure because they address the moving interface in its original form. Thus, we devised a simple change of variables that transforms the moving boundary problem to fixed boundary problem which in turn may be dealt with by virtually any standard numerical method.

The idea of the method is presented below. Let us assume that a system has $f$ phases $\left[\beta_{\alpha-1}, \beta_{\alpha}\right], \alpha=1, \ldots, f$. Concentration of $i$ th component in $\alpha$ phase will be denoted by $u_{i}^{\alpha}=u_{i}^{\alpha}(x, t)$. In this section an idea of numerical solution of Stefan-like problem given by Eq 12 is described.

\subsection{Equations}

$$
\left\{\begin{array}{l}
\frac{\partial u_{i}^{\alpha}}{\partial t}(x, t)=\frac{\partial}{\partial x}\left(D_{i}^{\alpha}\left(u_{i}^{\alpha}\right) \frac{\partial u_{i}^{\alpha}}{\partial x}(x, t)\right) \\
\quad \text { for } s_{\alpha-1}(t)<x<s_{\alpha}(t), \\
s_{\alpha}^{\prime}(t)=\sum_{i, j=1}^{r}\left(a_{i j}^{\alpha / \alpha+1} u_{i}^{\alpha}\left(s_{\alpha}(t), t\right) \frac{\partial u_{j}^{\alpha}}{\partial x}\left(s_{\alpha}(t), t\right)\right. \\
\left.\quad+a_{i j}^{\alpha+1 / \alpha} u_{i}^{\alpha+1}\left(s_{\alpha}(t), t\right) \frac{\partial u_{j}^{\alpha+1}}{\partial x}\left(s_{\alpha}(t), t\right)\right) \quad \text { for } t>0 .
\end{array}\right.
$$

where $a_{i j}^{\alpha-1 / \alpha}$ and $a_{i j}^{\alpha / \alpha+1}$ are known coefficients.

\subsection{Boundary Conditions}

Jumps of concentrations of an $i$ th component at $\alpha$ th boundary are denoted by $\delta_{i}^{\alpha}$.

Then

$$
\left\{\begin{array}{l}
u_{i}^{1}\left(s_{0}(t), t\right)=\delta_{i}^{1}, \\
u_{i}^{\alpha}\left(s_{\alpha}(t), t\right)=u_{i}^{\alpha+1}\left(s_{\alpha}(t), t\right)+\delta_{i}^{\alpha} \quad \text { for } 1 \leq \alpha \leq f-1, \\
u_{i}^{f}\left(s_{f}(t), t\right)=\delta_{i}^{f} .
\end{array}\right.
$$

\subsection{Initial Conditions}

Let us denote the initial positions of the phase boundaries by $\beta_{\alpha}$ for $\alpha=1, \ldots, f$. Then,

$\left\{\begin{array}{lr}u_{i}^{\alpha}(x, 0)=f_{i}^{\alpha}(x) & \text { for } x \in\left[\beta_{\alpha-1}, \beta_{\alpha}\right], \alpha=1, \ldots, f, \\ s_{\alpha}(0)=\beta_{\alpha} & \text { for } \alpha=0, \ldots, f .\end{array}\right.$

Such formulated problem is a moving boundary problem. For numerical computations, it is more convenient to transform the problem with moving boundaries into a problem with the fixed boundaries. This will be done by introducing suitable new variables.

Let us define the following auxiliary transformation $\varphi^{\alpha}$ :

$\varphi^{\alpha}:\left[\beta_{\alpha-1}, \beta_{\alpha}\right] \rightarrow\left[s_{\alpha-1}(t), s_{\alpha}(t)\right]$

$\varphi^{\alpha}(x, t)=s_{\alpha-1}(t)+\left(s_{\alpha}(t)-s_{\alpha-1}(t)\right) \frac{x-\beta_{\alpha-1}}{\beta_{\alpha}-\beta_{\alpha-1}}$

Using (15), we can define new variables:

$v_{i}^{\alpha}(x, t)=u_{i}^{\alpha}\left(\varphi^{\alpha}(x, t), t\right)$.

The suitable time and space derivatives of (16) are given as follows $\left(\varphi_{x}, \varphi_{t}\right.$ denote derivatives with respect to $x$ and $\left.t\right)$ :

$$
\begin{aligned}
& \frac{\partial v_{i}^{\alpha}}{\partial t}(x, t)=\frac{\partial u_{i}^{\alpha}}{\partial x}\left(\varphi^{\alpha}(x, t), t\right) \varphi_{t}^{\alpha}(x, t)+\frac{\partial u_{i}^{\alpha}}{\partial t}\left(\varphi^{\alpha}(x, t), t\right), \\
& \frac{\partial v_{i}^{\alpha}}{\partial x}(x, t)=\frac{\partial u_{i}^{\alpha}}{\partial x}\left(\varphi^{\alpha}(x, t), t\right) \varphi_{x}^{\alpha}(x, t), \\
& \frac{\partial v_{i}^{\alpha}}{\partial x}(x, t)=\frac{\partial^{2} u_{i}^{\alpha}}{\partial x^{2}}\left(\varphi^{\alpha}(x, t), t\right) \varphi_{x}^{\alpha}(x, t)
\end{aligned}
$$

(because $\varphi_{x}^{\alpha}$ does not depend on $x$ ),

$\varphi_{x}^{\alpha}(x, t)=\frac{s_{\alpha}(t)-s_{\alpha-1}(t)}{\beta_{\alpha}-\beta_{\alpha-1}}$,

$\varphi_{t}^{\alpha}(x, t)=s_{\alpha-1}^{\prime}(t)+\left(s_{\alpha}^{\prime}(t)-s_{\alpha-1}^{\prime}(t)\right) \frac{x-\beta_{\alpha-1}}{\beta_{\alpha}-\beta_{\alpha-1}}$.

In addition, the following relations hold:

$$
\begin{aligned}
& v_{i}^{\alpha}\left(\beta_{\alpha-1}, t\right)=u_{i}^{\alpha}\left(\varphi^{\alpha}\left(\beta_{\alpha-1}, t\right), t\right)=u_{i}^{\alpha}\left(s_{\alpha-1}(t), t\right), \\
& v_{i}^{\alpha}\left(\beta_{\alpha}, t\right)=u_{i}^{\alpha}\left(\varphi^{\alpha}\left(\beta_{\alpha}, t\right), t\right)=u_{i}^{\alpha}\left(s_{\alpha}(t), t\right), \\
& \frac{\partial v_{i}^{\alpha}}{\partial x}\left(\beta_{\alpha-1}, t\right)=\frac{\partial u_{i}^{\alpha}}{\partial x}\left(s_{\alpha-1}(t), t\right) \varphi_{x}^{\alpha}(x, t), \\
& \frac{\partial v_{i}^{\alpha}}{\partial x}\left(\beta_{\alpha}, t\right)=\frac{\partial u_{i}^{\alpha}}{\partial x}\left(s_{\alpha-1}(t), t\right) \varphi_{x}^{\alpha}(x, t) .
\end{aligned}
$$

Using the above formulas, problems (12)-(14) can be transformed as shown below. For simplicity of presentation, we show calculations only for constant diffusion coefficients (full form, i.e., for concentration-dependent diffusivities as given in Appendix 1): 


$$
\begin{aligned}
\frac{\partial v_{i}^{\alpha}}{\partial t}(x, t) & =\frac{\partial u_{i}^{\alpha}}{\partial t}\left(\varphi^{\alpha}(x, t), t\right)+\frac{\partial u_{i}^{\alpha}}{\partial x}\left(\varphi^{\alpha}(x, t), t\right) \varphi_{t}^{\alpha}(x, t) \\
& =\frac{\partial^{2} u_{i}^{\alpha}}{\partial x^{2}}\left(\varphi^{\alpha}(x, t), t\right)+\frac{\partial u_{i}^{\alpha}}{\partial x}\left(\varphi^{\alpha}(x, t), t\right) \varphi_{t}^{\alpha}(x, t) \\
& =D_{i}^{\alpha} \frac{1}{\varphi_{x}^{\alpha}} \frac{\partial^{2} v_{i}^{\alpha}}{\partial x^{2}}(x, t)+\frac{\varphi_{t}^{\alpha}}{\varphi_{x}^{\alpha}} \frac{\partial v_{i}^{\alpha}}{\partial x}(x, t),
\end{aligned}
$$

and

$$
\left\{\begin{aligned}
\frac{\partial v_{i}^{\alpha}}{\partial t}(x, t) & =D_{i}^{\alpha} \frac{1}{\varphi_{x x}^{\alpha}} \frac{\partial^{2} v_{i}^{\alpha}}{\partial x^{2}}(x, t)+\frac{\varphi_{t}^{\alpha}}{\varphi_{x}^{\alpha}} \frac{\partial v_{i}^{\alpha}}{\partial x}(x, t), \\
s_{\alpha}^{\prime}(t)= & \sum_{i, j=1}^{r}\left(\frac{a_{i j}^{\alpha / \alpha+1}}{\varphi_{x}^{\alpha}} v_{i}^{\alpha}\left(\beta_{\alpha}, t\right) \frac{\partial v_{j}^{\alpha}}{\partial x}\left(\beta_{\alpha}, t\right)\right. \\
& \left.+\frac{a_{i j}^{\alpha+1 / \alpha}}{\varphi_{x}^{\alpha+1}} v_{i}^{\alpha+1}\left(\beta_{\alpha}, t\right) \frac{\partial u_{j}^{\alpha+1}}{\partial x}\left(s_{\alpha}(t), t\right)\right)
\end{aligned}\right.
$$

Taking into account Eq 17, we can write

$$
\left\{\begin{aligned}
\frac{\partial v_{i}^{\alpha}}{\partial t}(x, t) & =\frac{\beta_{\alpha}-\beta_{\alpha-1}}{s_{\alpha}(t)-s_{\alpha-1}(t)}\left(D_{i}^{\alpha} \frac{\partial^{2} v_{i}^{\alpha}}{\partial x^{2}}(x, t)\right. \\
& \left.+s_{\alpha-1}^{\prime}(t)+\left(s_{\alpha}^{\prime}(t)-s_{\alpha-1}^{\prime}(t)\right) \frac{x-\beta_{\alpha-1}}{\beta_{\alpha}-\beta_{\alpha-1}} \frac{\partial v_{i}^{\alpha}}{\partial x}(x, t)\right), \\
s_{\alpha}^{\prime}(t)= & \sum_{i, j=1}^{r}\left(a_{i j}^{\alpha / \alpha+1} \frac{\beta_{\alpha}-\beta_{\alpha-1}}{s_{\alpha}(t)-s_{\alpha-1}(t)} v_{i}^{\alpha}\left(\beta_{\alpha}, t\right) \frac{\partial v_{j}^{\alpha}}{\partial x}\left(\beta_{\alpha}, t\right)\right. \\
& \left.+a_{i j}^{\alpha+1 / \alpha} \frac{\beta_{\alpha+1}-\beta_{\alpha}}{s_{\alpha+1}(t)-s_{\alpha}(t)} v_{i}^{\alpha+1}\left(\beta_{\alpha}, t\right) \frac{\partial u_{j}^{\alpha+1}}{\partial x}\left(s_{\alpha}(t), t\right)\right) .
\end{aligned}\right.
$$

Boundary conditions (14) in the fixed boundary problem have the following forms:

$$
\left\{\begin{array}{l}
v_{i}^{\alpha}\left(\beta_{0}, t\right)=\delta_{i}^{1}, \\
v_{i}^{\alpha}\left(\beta_{\alpha}, t\right)=v_{i}^{\alpha+1}\left(\beta_{\alpha}, t\right)+\delta_{i}^{\alpha} \quad \text { for } 1 \leq \alpha \leq f-1, \\
v_{i}^{f}\left(\beta_{f}, t\right)=\delta_{i}^{f},
\end{array}\right.
$$

and the corresponding initial conditions (14) read as

$$
\left\{\begin{array}{l}
v_{i}^{\alpha}(x, 0)=f_{i}^{\alpha}(x) \quad \text { for } x \in\left[\beta_{\alpha-1}, \beta_{\alpha}\right], \quad \alpha=1, \ldots, f, \\
s_{\alpha}(0)=\beta_{\alpha} \quad \text { for } \alpha=0, \ldots, f .
\end{array}\right.
$$

\subsection{Multi-Phase Binary System}

In this section, the case of diffusion soldering process in binary system $(\mathrm{Ag} / \mathrm{Sn} / \mathrm{Ag})$ is presented. We consider binary system with multiple phases. Following the Darken drift idea (Ref 16), such system may be described by one concentration, $c^{\alpha}(x, t)$, and one diffusion coefficient, $\tilde{D}^{\alpha}$, in each phase (the chemical diffusion coefficient).

$$
\left\{\begin{aligned}
\frac{\partial c^{\alpha}}{\partial t}(x, t)= & \frac{\partial}{\partial x}\left(\tilde{D}^{\alpha} \frac{\partial c^{\alpha}}{\partial x}(x, t)\right) \\
s_{\alpha-1}(t)<x< & s_{\alpha}(t), \\
\left.\left(c^{\alpha+1}-c^{\alpha}\right)\right|_{x=s^{\alpha}(t)} s_{\alpha}^{\prime}(t) & =-\tilde{D}^{\alpha+1} \frac{\partial c^{\alpha+1}}{\partial x}\left(s^{\alpha}(t), t\right) \\
& +\tilde{D}^{\alpha} \frac{\partial c^{\alpha}}{\partial x}\left(s^{\alpha}(t), t\right), \quad t>0,
\end{aligned}\right.
$$

where $\alpha=1, \ldots, f$.
In general, the chemical diffusion coefficient may be variable. However, to verify the model with experiment, we assumed $\tilde{D}^{\alpha}=$ const.

Using the same type of change of variables as in (16), i.e., $v^{\alpha}(x, t)=c^{\alpha}\left(\varphi^{\alpha}(x, t), t\right)$, and following virtually the same calculations as in (17)-(20), we arrive at

$$
\left\{\begin{aligned}
\frac{\partial \nu^{\alpha}}{\partial t}(x, t) & =\frac{\beta_{\alpha}-\beta_{\alpha-1}}{s_{\alpha}(t)-s_{\alpha-1}(t)}\left(\tilde{D}^{\alpha} \frac{\partial^{2} v^{\alpha}}{\partial x^{2}}(x, t)\right. \\
& \left.+s_{\alpha-1}^{\prime}(t)+\left(s_{\alpha}^{\prime}(t)-s_{\alpha-1}^{\prime}(t)\right) \frac{x-\beta_{\alpha-1}}{\beta_{\alpha}-\beta_{\alpha-1}} \frac{\partial v^{\alpha}}{\partial x}(x, t)\right), \\
& \beta_{\alpha-1}<x<\beta_{\alpha}, \\
s_{\alpha}^{\prime}(t)= & \tilde{D}^{\alpha+1} \frac{\beta_{\alpha}-\beta_{\alpha-1}}{s_{\alpha}(t)-s_{\alpha-1}(t)} \frac{\partial v^{\alpha}}{\partial x}\left(\beta_{\alpha}, t\right) \\
- & \tilde{D}^{\alpha} \frac{\beta_{\alpha+1}-\beta_{\alpha}}{s_{\alpha+1}(t)-s_{\alpha}(t)} \frac{\partial \nu^{\alpha+1}}{\partial x}\left(s_{\alpha}(t), t\right), t>0 .
\end{aligned}\right.
$$

\subsection{Numerical Solution}

Original moving boundary problem (23) was transformed into fixed boundary problem (24) that can be solved numerically using standard methods, e.g., method of lines (Ref 17, 18). Space derivatives only have been discretized using secondorder scheme (see Appendix 1). Consequently, initial boundary-value problem for partial differential equations has been transformed into the initial (Cauchy) problem for ordinary differential equations (ODEs):

$$
\left\{\begin{aligned}
\frac{d v_{k}^{\alpha}}{d t}= & \tilde{D}^{\delta} \frac{\beta_{\alpha}-\beta_{\alpha-1}}{s^{\alpha}(t)-s^{\alpha-1}(t)} \frac{v_{k+1}^{\alpha}+v_{k-1}^{\alpha}-2 v_{k}^{\alpha}}{h_{\alpha}^{2}}+\frac{\beta_{\alpha}-\beta_{\alpha-1}}{s^{\alpha}(t)-s^{\alpha-1}(t)} \\
& \left(s_{\alpha-1}^{\prime}(t)+\left(s_{\alpha}^{\prime}(t)-s_{\alpha-1}^{\prime}(t)\right) \frac{k h_{\alpha}}{\beta_{\alpha}-\beta_{\alpha-1}}\right) . \\
& \cdot \frac{v_{k+1}^{\alpha}-v_{k-1}^{\alpha}}{2 h_{\alpha}}, \quad k=1, \ldots, n-1, \\
s_{\alpha}^{\prime}(t)= & \frac{\tilde{D}^{\alpha}}{\delta_{1}} \frac{\beta_{\alpha}-\beta_{\alpha-1}}{s^{\alpha}(t)-s^{\alpha-1}(t)} \\
& \cdot\left[\frac{-3 v_{0}^{\alpha+1}+4 v_{1}^{\alpha+1}-v_{2}^{\alpha+1}}{2 h_{\alpha+1}}-\frac{v_{n-2}^{\alpha}-4 v_{n-1}^{\alpha}+3 v_{n}^{\alpha}}{2 h_{\alpha}}\right] \\
v_{k}^{\alpha}(0)= & v_{k}^{\alpha, 0} \text { for } k=0, \ldots, n ; s_{\alpha}(0)=\beta_{\alpha} .
\end{aligned}\right.
$$

where $\delta_{\alpha}=\left.\left(c^{\alpha+1}-c^{\alpha}\right)\right|_{x=s^{\alpha}(t)}$ is the jump of concentration at the $(\alpha-1 \mid \alpha)$ boundary. This value may be calculated from the binary phase diagram, and as such it is considered here as a known value.

The system (25) has been numerically solved using the Radau II method for stiff system of equations (Ref 19).

\subsection{Test Problem with Analytic Solution}

For the test of numerical procedure, we take the well-known problem with moving boundary which admits analytic expression for the boundary movement. A phase $\alpha$ grows from homogeneous semi-infinite phase $\gamma$ in a binary alloy. The initial concentration of the phase $\gamma$ is denoted by $c^{\infty}$. The local mass balance (10) now reads 
$\left(c^{\gamma}-c^{\alpha}\right) s^{\prime}(t)=J^{\gamma}-J^{\alpha}$,

but we assume that the concentration in the phase $\alpha$ is constant; thus $J^{\alpha}=0$. The flux $J^{\gamma}$ is governed by the standard Fick's law, $J^{\gamma}=-D^{\gamma}\left(\partial^{2} c^{\gamma}\right) /\left(\partial x^{2}\right)$. The analytic solution to this problem can be found, for example, in Ref 4. It states that the phase boundary $s=s(t)$ is moving according to parabolic law:

$s(t)=K \sqrt{D^{\gamma} t}$,

where the growth constant $K$ is the solution of the following nonlinear equation:

$K=\frac{2}{\sqrt{\pi}} \Omega \frac{e^{-K^{2} / 4}}{1-\operatorname{erf}(K / 2)}$,

with dimensionless parameter

$\Omega=\frac{c^{\gamma}-c^{\infty}}{c^{\gamma}-c^{\alpha}}$

The numerical solution to the test problem is presented in Appendix 2. In Fig. 1, the thickness of the growing phase as a function of time, $s(t)$, for various values of parameter $\Omega$ are presented. Results obtained on the proposed numerical method corresponds well with analytic solution Eq 27-29.

\section{Experiment}

In order to verify the model presented above, the results of the examinations on the diffusion modeling in $\mathrm{Ag} / \mathrm{Sn} / \mathrm{Ag}$ system were taken (Ref 20). In this experiment, two pieces of pure silver were soldered using $\mathrm{Sn}$ as an interlayer. All the details concerning sample preparation and diffusion soldering process are presented elsewhere (Ref 20). The experiments were performed at the following conditions (see Table 1).

The obtained $\mathrm{Ag} / \mathrm{Sn} / \mathrm{Ag}$ interconnectors were characterized using light and scanning electron microscopy (SEM) techniques, while the $\mathrm{Sn}$ concentration profiles across the joints were determined using the energy x-ray dispersive spectroscopy (EDX) (for details see also Ref 20).

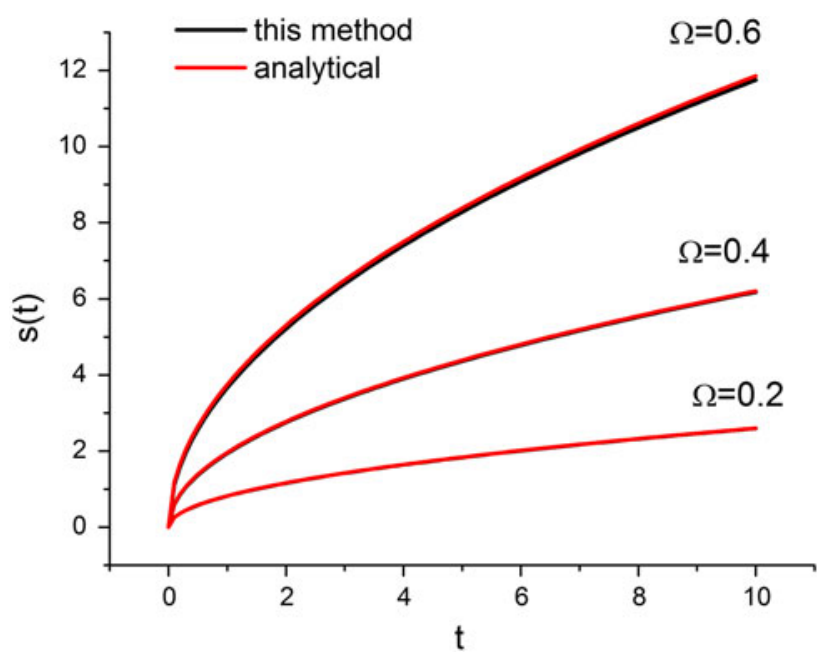

Fig. 1 Comparison between an analytic solution (red lines) and numerical method (black lines) for the test Stefan problem (Color figure online)
Typical examples of microstructures after the diffusion soldering process at the temperatures 243 and $258{ }^{\circ} \mathrm{C}$ are shown in Fig. 2.

As example, Sn composition profiles of the elements across the joint are presented in Fig. 3.

\subsection{Kinetics}

For the description of the kinetics of intermetallic phase growth, $d(t)$, for diffusion soldering process, the following equation has been used:

$d(t)=k \cdot t^{n}$.

If the value of the kinetic parameter is $n=0.5$, then this corresponds to the volume diffusion, while for range $n<0.5$, one can expect some contribution from the grain boundary diffusion.

Table 1 Production conditions of diffusion joints of the Ag/Sn/Ag type

\begin{tabular}{ll}
\hline Temperature, ${ }^{\circ} \mathbf{C}$ & \multicolumn{1}{c}{ Time, $\min$} \\
\hline 230 & $10,30,60,90,120,150$ \\
243 & $10,20,30,40,60,80$ \\
250 & $10,20,60,120,180$ \\
258 & $20,30,40,50,60,80$ \\
265 & $10,20,30,60,90,120$ \\
\hline
\end{tabular}
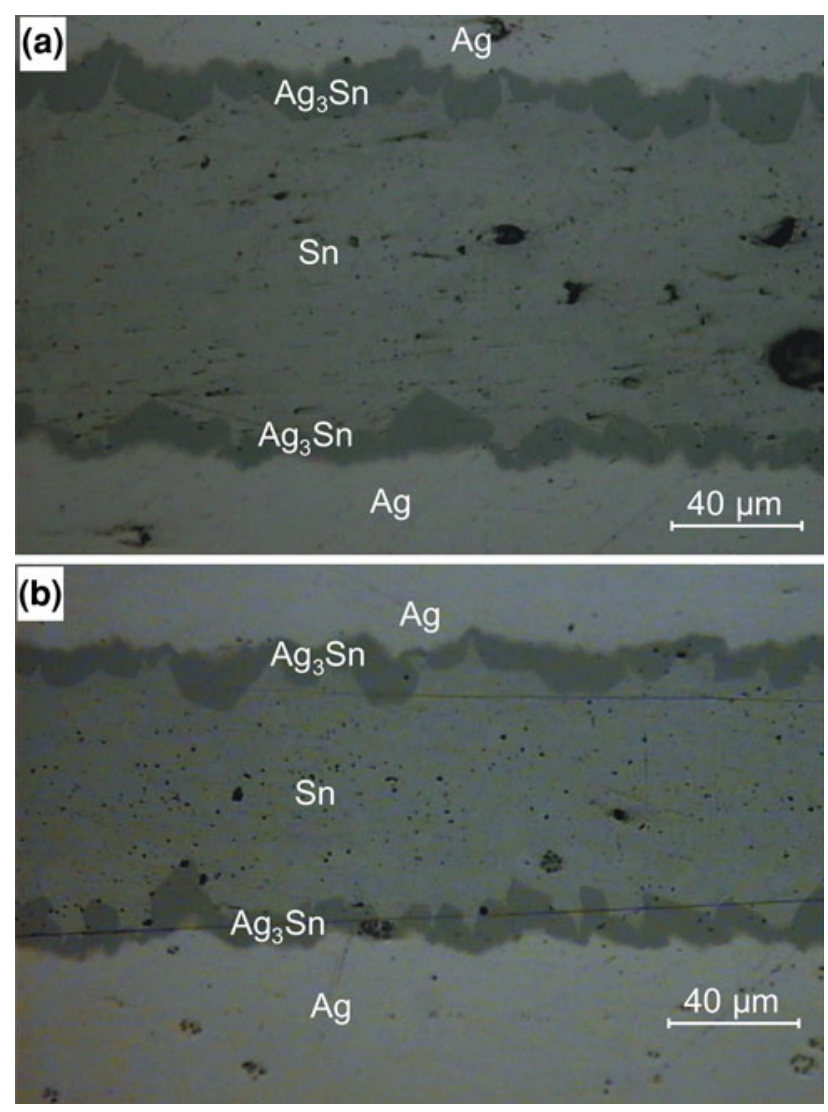

Fig. 2 Light microscope image of diffusion soldering joint $\mathrm{Ag} / \mathrm{Sn}$ / $\mathrm{Ag}$ after annealing: (a) $243^{\circ} \mathrm{C}$ for $80 \mathrm{~min}$ and (b) $258^{\circ} \mathrm{C}$ for $50 \mathrm{~min}$ 
In order to determine the kinetic parameter (exponential factor) $n$, the thickness of $\mathrm{Ag}_{3} \mathrm{Sn}$ intermetallic phase was measured after various periods of time (see Table 1) applying the following procedure.

Because of an uneven and rugged growth of intermetallic phases along the joint, a specialized computer program was used to evaluate the width of the selected phase. The program probes the width in 600 points over a specified area and calculates the average width and standard deviation (Fig. 4).

Four to six measurements were carried out for each intermetallic phase on both sides of the joint in different and representative areas of the sample keeping both sides parallel. Hence, no correction of the measurements due to the slope of the sample with respect to the optical axis of a microscope was necessary. In addition, the locations where the intermetallic phases grew in the form of scallops were not taken into account which helped us to avoid larger deviations for individual measurements. Hence, each calculated result is the average from many measurements, and the error produced by the

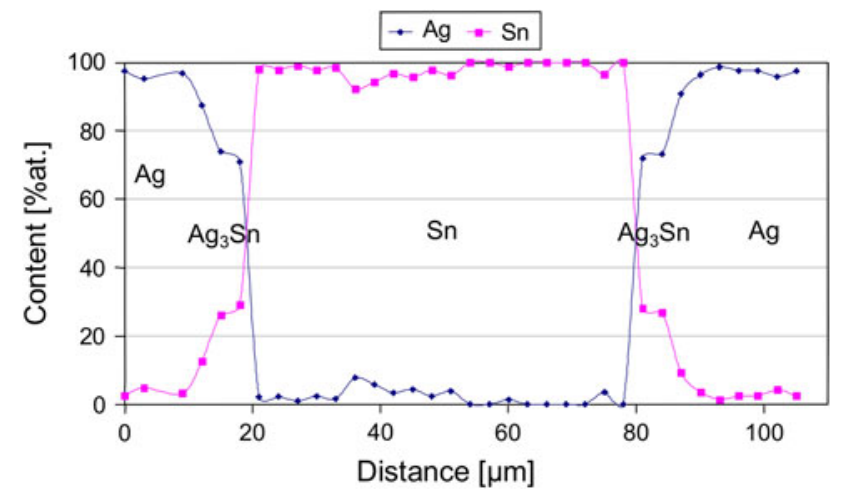

Fig. 3 EDX linescan with $3 \mu \mathrm{m}$ step across $\mathrm{Ag} / \mathrm{Sn} / \mathrm{Ag}$ joint at temperature $243{ }^{\circ} \mathrm{C}$ for $20 \mathrm{~min}$ (with permission from Archives of Metallurgy and Materials (Ref 20)) program may have slightly different values even for the same series of samples.

Figure 7 presents measured $\mathrm{Ag}_{3} \mathrm{Sn}$ phase thicknesses $d(t)$ vs. the annealing time $t$ for different temperatures. The resulting values of kinetic parameter $n$ in the Eq 30 were determined on the basis of $\log d$ vs. $\log t$ plots, and are listed in Table 2 .

In the temperature range of $230-243{ }^{\circ} \mathrm{C}$, a clear decrease of the $n$ value is visible indicating the change of the diffusion mechanism, which can be explained by the growing contribution of the grain boundary diffusion and the decrease of the phase boundary reaction contribution up to it, leading to the $\mathrm{Ag}_{3} \mathrm{Sn}$ phase formation. At the temperature of $250{ }^{\circ} \mathrm{C}$, an anomalous decrease of the $n$ value was noticed, which, however, cannot be clearly explained by the present research. At $265{ }^{\circ} \mathrm{C}$, the $n$ value increases again, and it may indicate the volume diffusion as the rate controlling factor.

\subsection{Modeling of Reactive Diffusion in $\mathrm{Ag} / \mathrm{Sn} / \mathrm{Ag}$ Interconnectors}

Modeling of intermetallic phase $\left(\mathrm{Ag}_{3} \mathrm{Sn}\right)$ growth for different temperatures in $\mathrm{Ag} / \mathrm{Sn} / \mathrm{Ag}$ joints have been performed based on the presented model. Proposed numerical method allowed us to solve effectively the problem. For calculations, the following data have been used:

Table 2 The values of the kinetic parameter, $n$, for $\mathrm{Ag}_{3} \mathrm{Sn}$ phase growth for different temperatures of the $\mathrm{Ag} / \mathrm{Sn} / \mathrm{Ag}$ joint

\begin{tabular}{lc}
\hline Temperature, ${ }^{\circ} \mathbf{C}$ & Parameter, $\boldsymbol{n}$ \\
\hline 230 & $0.67 \pm 0.10$ \\
243 & $0.38 \pm 0.07$ \\
250 & $0.17 \pm 0.02$ \\
258 & $0.45 \pm 0.07$ \\
265 & $0.55 \pm 0.02$ \\
\hline
\end{tabular}
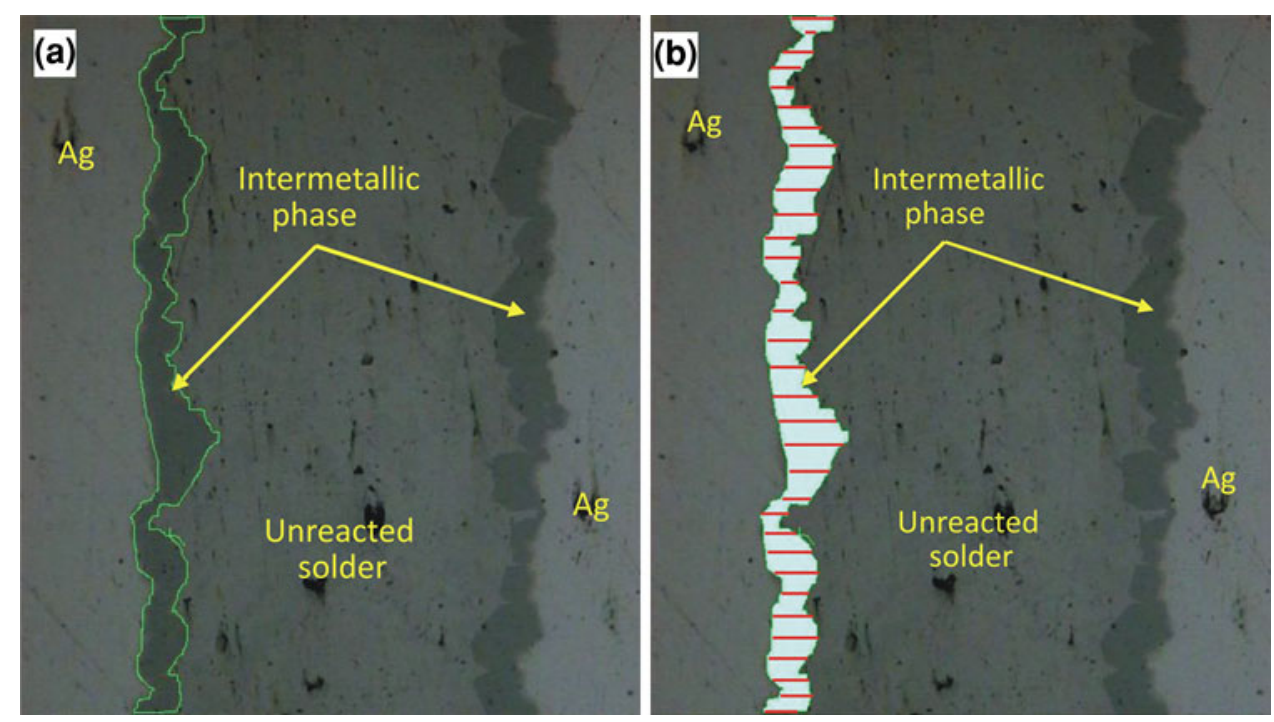

Fig. 4 Example images of selected area for computation of the average intermetallic phase width (a), and the schema showing how the width probing was performed to assess the intermetallic width in a joint obtain by the low-temperature diffusion soldering (b). The red lines indicate the locations where the measurements were carried out by the program (Color figure online) 
Data

(1) $c_{\mathrm{Sn}, \mathrm{R}}^{(\mathrm{Ag})}=0.1012 \mathrm{~mol} / \mathrm{mol}$

(2) $c_{\mathrm{Sn}, \mathrm{L}}^{\varepsilon}=0.2295 \mathrm{~mol} / \mathrm{mol}$

(3) $\quad c_{\mathrm{Sn,R}}^{\varepsilon}=0.2476 \mathrm{~mol} / \mathrm{mol}$

(4) $\quad c_{\mathrm{Sn} \mathrm{L}}^{\mathrm{Ln} \mathrm{R}}=0.95 \mathrm{~mol} / \mathrm{mol}$

(5) $\beta_{1} \stackrel{\text { L }}{=}, \beta_{1}=1 \times 10^{-9} \mathrm{~m}$

(6) Calculated $\tilde{D}^{\varepsilon}= \begin{cases}230^{\circ} \mathrm{C} & 3.9 \times 10^{-13} \mathrm{~m}^{2} / \mathrm{s} \\ 243^{\circ} \mathrm{C} & 6.3 \times 10^{-13} \mathrm{~m}^{2} / \mathrm{s} \\ 250^{\circ} \mathrm{C} & 1.1 \times 10^{-13} \mathrm{~m}^{2} / \mathrm{s} \\ 258^{\circ} \mathrm{C} & 8.2 \times 10^{-13} \mathrm{~m}^{2} / \mathrm{s} \\ 265^{\circ} \mathrm{C} & 1.4 \times 10^{-12} \mathrm{~m}^{2} / \mathrm{s}\end{cases}$

(7) $N=200$-number of nodes in the approximation scheme.

Explanation of the symbols used above is presented in the Fig. 5.

The calculated diffusion coefficients have been presented in Arrhenius plot (Fig. 6). Except for $250{ }^{\circ} \mathrm{C}$, the points can be successfully approximated by the linear function. At $250{ }^{\circ} \mathrm{C}$, the obtained parameter $n$ was found to be 0.17 . Such a value corresponds to the significant contribution coming from grain boundary diffusion (Ref 21 ). The calculated activation energy equals $79 \pm 13 \mathrm{~kJ} / \mathrm{mol}$. Similar value of $70.3 \mathrm{~kJ} / \mathrm{mol}$ was obtained by $\mathrm{Su}$ et al. (Ref 22). Flanders et al. (Ref 23) obtained activation energy in $\mathrm{Cu} / \mathrm{Sn}-\mathrm{Ag} / \mathrm{Cu}$ system for phase $\mathrm{Cu}_{3} \mathrm{Sn}$ to be $70.7 \mathrm{~kJ} / \mathrm{mol}$.

In Fig. 7, $\mathrm{Ag}_{3} \mathrm{Sn}$ intermetallic phase width is calculated as a function of time for different temperatures and compared with experimental results showing satisfactory agreement. The calculated kinetics of intermetallic phase growth shows parabolic behavior. This is the consequence of simplifying assumption that the thickness of outer phases being in contact with intermetallic $\mathrm{Ag}_{3} \mathrm{Sn}$ phase is semi-infinite. It ensures that concentrations at the boundaries are constant. This is not the limitation of the model, but we selected this semi-infinite configuration based on experimental results - see, EDX concentration profiles taken across the joint (Fig 3).

An example of more complex configuration which leads to non-parabolic diffusion growth is illustrated in Fig. 8 and 9.

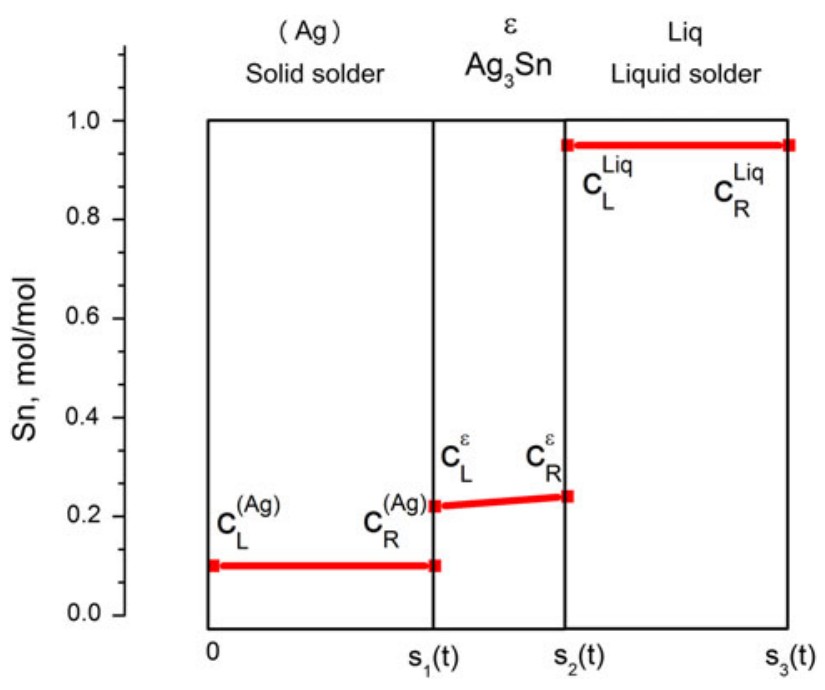

Fig. 5 Model of $\mathrm{Ag}_{3} \mathrm{Sn}$ intermetallic phase growth during diffusion soldering process
The calculations were performed for the system with four moving boundaries and the following data:

(1) Initial positions of the boundaries $s_{i}(0)=\beta_{i}=\{0,0.01$, $0.02,30\} \times 10^{-6} \mathrm{~m}$

(2) Diffusion coefficients in each layer $\left[s_{i}(t), s_{i+1}(t)\right]$, $D_{i}=\{5,1,0.1\} \times 10^{-13} \mathrm{~m}^{2} / \mathrm{s}$

Figure 8 shows evolution of moving boundaries $s_{i}(t)$, and Fig. 9 shows thicknesses of layers, $d_{i}(t)=s_{i+1}(t)-s_{i}(t)$, vs. time in a parabolic plot.

It can be seen that thicknesses of the layers exhibit initial non-parabolic growth. In particular, the first layer, $d_{1}(t)$, is monotonically increasing, the third one, $d_{3}(t)$, is monotonically decreasing, and the second, $d_{2}(t)$, shows non-monotonic evolution.

\section{Conclusions}

The mathematical model describing reactive diffusion in multi-component system with moving boundaries has been presented. Effective numerical method to solve the problem has been developed and applied. In the numerical approach, a moving boundary problem was converted into the equivalent fixed boundary problem. The resulting partial differential equations system was treated by the method of lines which gives a system of ordinary equations, which was subsequently solved numerically by the Radau II method. The investigation of the growth kinetics of intermetallic $\mathrm{Ag}_{3} \mathrm{Sn}$ phase during diffusion soldering revealed different diffusion mechanisms. The $\mathrm{Ag}_{3} \mathrm{Sn}$ phase grew as a product of the volume diffusion when kinetic parameter $n$ is close to 0.5 . In this case, a good agreement between calculated and experimental results has been obtained. When $n=0.17\left(T=250^{\circ} \mathrm{C}\right)$, the significant deviation from the slope of Arrhenius plot was observed which indicates that the model cannot be applied. In the case of strong contribution of grain boundary diffusion, generalized boundary conditions including non-planar geometry of the interface (2D and/or 3D models) are necessary to describe formation of "scallops." Such model will be subject of a separate article.

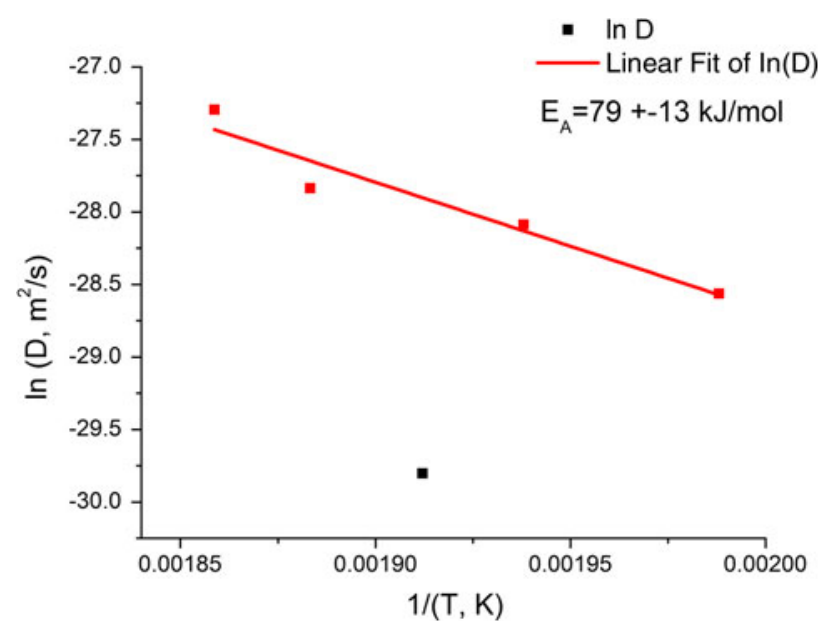

Fig. 6 Diffusion coefficients for $\mathrm{Ag}_{3} \mathrm{Sn}$ intermetallic phase as a function temperature in the Arrhenius plot 

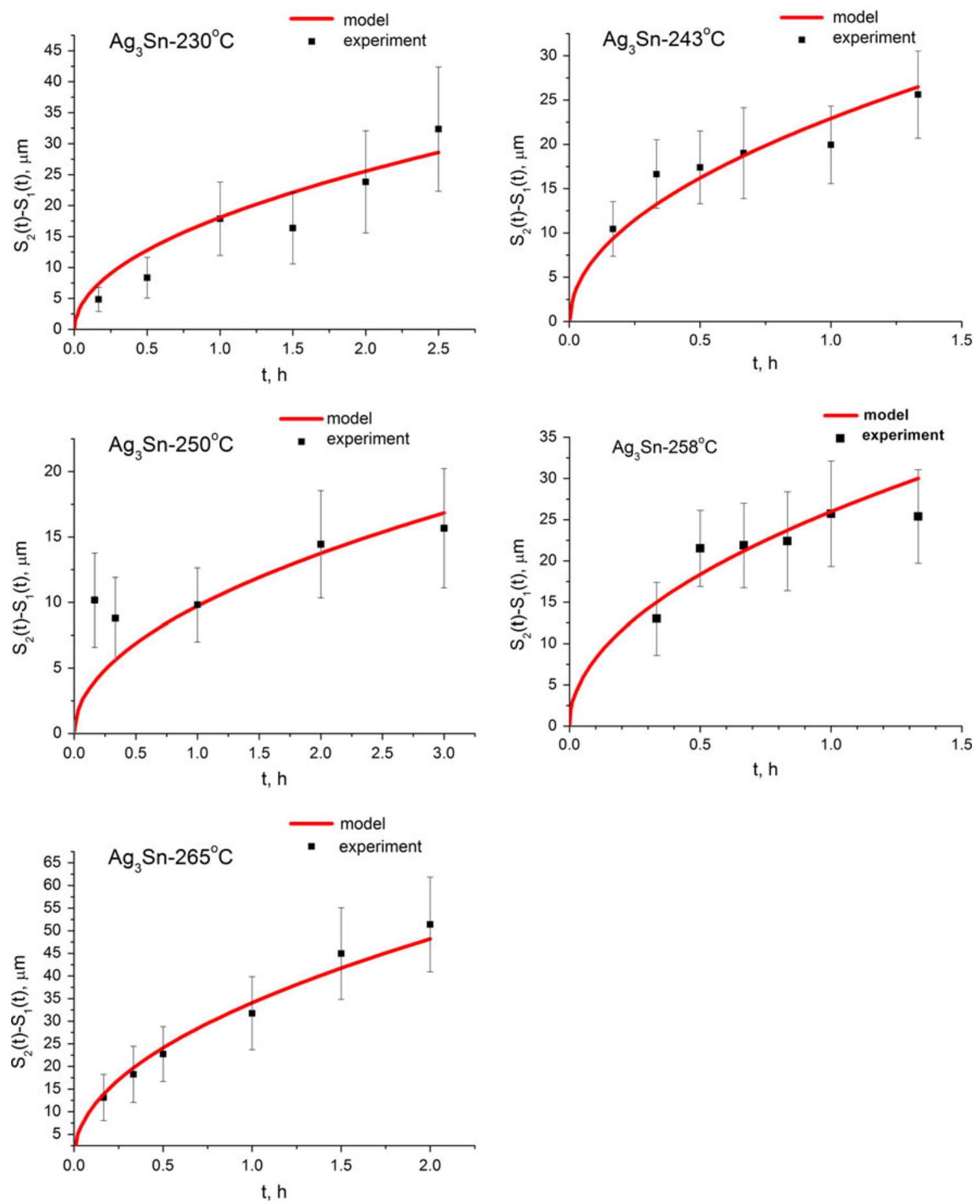

Fig. 7 Calculated width of $\mathrm{Ag}_{3} \mathrm{Sn}$ as a function of time for temperatures: 230, 243, 250, 258 and $265{ }^{\circ} \mathrm{C}$. Comparison with experimental results

\section{Acknowledgment}

This study has been supported by the Polish Ministry of Higher Education and Science-AGH grant no. 11.11.160.800.

\section{Open Access}

This article is distributed under the terms of the Creative Commons Attribution License which permits any use, distribu-

tion, and reproduction in any medium, provided the original author(s) and the source are credited.

\section{Appendix 1: Fixed Boundary Reformulation for Concentration-Dependent Diffusivities}

By chain rule, we have from (12) 


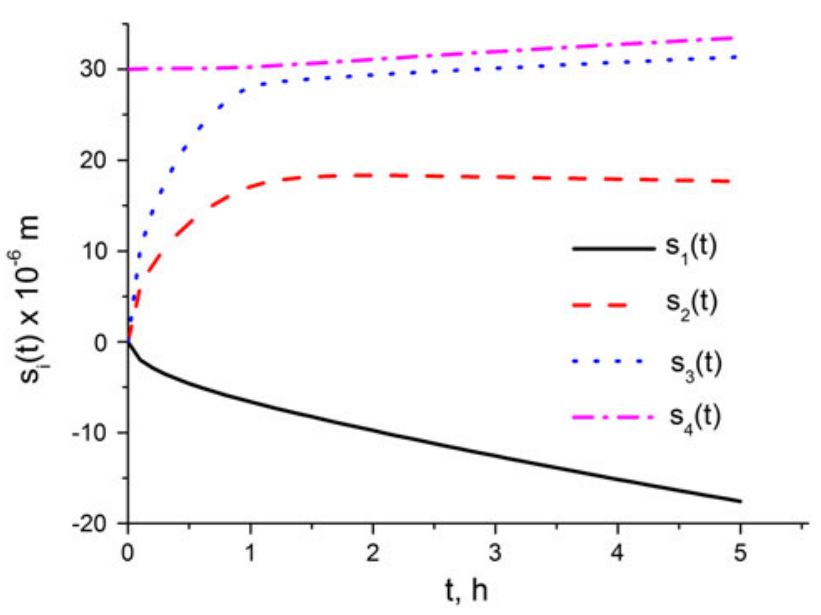

Fig. 8 Positions of the boundaries as function of time

$\frac{\partial u_{i}^{\alpha}}{\partial t}(x, t)=D\left(u_{i}^{\alpha}(x, t)\right) \frac{\partial^{2} u_{i}^{\alpha}}{\partial x^{2}}(x, t)+D^{\prime}\left(u_{i}^{\alpha}(x, t)\right)\left(\frac{\partial u_{i}^{\alpha}}{\partial x}(x, t)\right)^{2}$.

Substituting $v_{i}^{\alpha}(x, t)=u_{i}^{\alpha}\left(s_{\alpha}(t) x, t\right)$ into this equation and using notation $E=d D / d u$ after some calculation, we obtain (31). More details on this derivation are provided in (15)-(22).

$$
\left\{\begin{aligned}
\frac{\partial v_{i}^{\alpha}}{\partial t}(x, t)= & \frac{\beta_{\alpha}-\beta_{\alpha-1}}{s_{\alpha}(t)-s_{\alpha-1}(t)}\left(D_{i}^{\alpha}\left(v_{i}^{\alpha}\right) \frac{\partial^{2} v_{i}^{\alpha}}{\partial x^{2}}(x, t)+s_{\alpha-1}^{\prime}(t)\right. \\
& +\left(s_{\alpha}^{\prime}(t)-s_{\alpha-1}^{\prime}(t)\right) \frac{x-\beta_{\alpha-1}}{\beta_{\alpha}-\beta_{\alpha-1}} \frac{\partial v_{i}^{\alpha}}{\partial x}(x, t) \\
& \left.+E\left(v_{i}^{\alpha}\right)\left(\frac{\partial v_{i}^{\alpha}}{\partial x}(x, t)\right)^{2}\right) \\
s_{\alpha}^{\prime}(t)= & \sum_{i, j=1}^{r}\left(a_{i j}^{\alpha / \alpha+1} \frac{\beta_{\alpha}-\beta_{\alpha-1}}{s_{\alpha}(t)-s_{\alpha-1}(t)} v_{i}^{\alpha}\left(\beta_{\alpha}, t\right) \frac{\partial v_{j}^{\alpha}}{\partial x}\left(\beta_{\alpha}, t\right)\right. \\
+ & \left.a_{i j}^{\alpha+1 / \alpha} \frac{\beta_{\alpha+1}-\beta_{\alpha}}{s_{\alpha+1}(t)-s_{\alpha}(t)} v_{i}^{\alpha+1}\left(\beta_{\alpha}, t\right) \frac{\partial v_{j}^{\alpha+1}}{\partial x}\left(s_{\alpha}(t), t\right)\right) \\
x \in & {\left[\beta_{\alpha-1}, \beta_{\alpha}\right], \alpha=1, \ldots, f . }
\end{aligned}\right.
$$

\section{Appendix 2: Space Discretization on an Even Grid

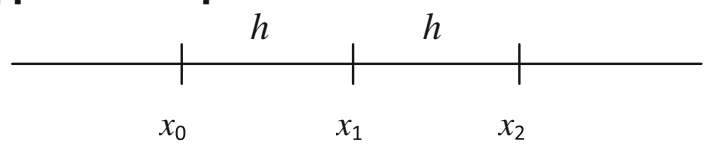

The First Derivative Inside

Let $f \in C^{2}([a, b] ; R)$. Then, we have

$$
f^{\prime}\left(x_{1}\right)=\frac{f\left(x_{2}\right)-f\left(x_{0}\right)}{2 h}+r(h)
$$

where $r(h)=-1 / 6\left(f^{\prime \prime \prime}\left(\xi_{0}\right)+f^{\prime \prime \prime}\left(\xi_{1}\right)\right) h^{2}$ for some $\left.\xi_{0} \in\right] x_{0}, x_{1}[$, $\left.\xi_{1} \in\right] x_{1}, x_{2}[$.

\section{The Second Derivative Inside}

Let $f \in C^{3}([a, b] ; R)$. Then, we have

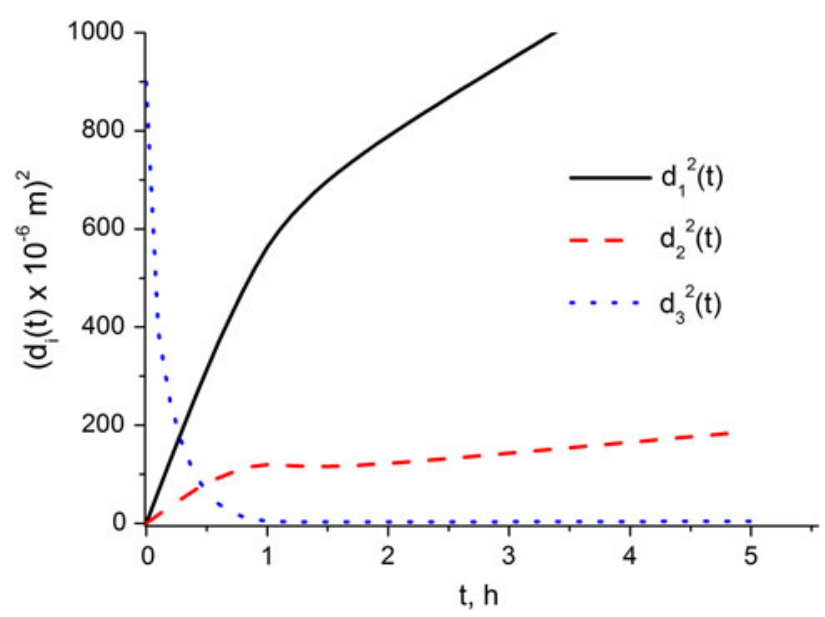

Fig. 9 Thicknesses of layers as function of time - parabolic plot

$$
f^{\prime \prime}\left(x_{1}\right)=\frac{f\left(x_{0}\right)-2 f\left(x_{1}\right)+f\left(x_{2}\right)}{h^{2}}+r(h)
$$

where $r(h)=-1 / 24\left(f^{(4)}\left(\xi_{0}\right)+f^{(4)}\left(\xi_{1}\right)\right) h^{2}$ for some $\left.\xi_{0} \in\right] x_{0}$, $x_{1}\left[, \xi_{1} \in\right] x_{1}, x_{2}[$.

\section{The First Derivative at the Boundaries}

Left Boundary. We may use the set of the points as above, but this time, we want to approximate a derivative $f\left(x_{0}\right)$ at $x_{0}$ by the values of $f$ at the points $x_{1}, x_{2}$, which lie to the right of $x_{0}$. Using two Taylor expansions at $x_{0}$, we can easily calculate

$$
f^{\prime}\left(x_{0}\right)=\frac{-3 f\left(x_{0}\right)+4 f\left(x_{1}\right)-f\left(x_{2}\right)}{2 h}+r(h)
$$

where $r(h)=1 / 3\left(2 f^{\prime \prime \prime}\left(\xi_{1}\right)-f^{\prime \prime \prime}\left(\xi_{0}\right)\right) h^{2}$ for some $\left.\xi_{0} \in\right] x_{0}, x_{1}[$, $\left.\xi_{1} \in\right] x_{1}, x_{2}[$.

Right Boundary. Now we want to approximate a derivative $f\left(x_{2}\right)$ at $x_{2}$ by the values of $f$ at the points $x_{0}, x_{1}$, which lie to the left of $x_{2}$. Using two Taylor expansions at $x_{2}$, we can easily derive

$$
f^{\prime}\left(x_{2}\right)=\frac{f\left(x_{0}\right)-4 f\left(x_{1}\right)+3 f\left(x_{2}\right)}{2 h}+r(h) .
$$

where $r(h)=1 / 3\left(2 f^{\prime \prime \prime}\left(\xi_{1}\right)-f^{\prime \prime \prime}\left(\xi_{0}\right)\right) h^{2}$ for some $\left.\xi_{0} \in\right] x_{0}, x_{1}$ $\left[, \xi_{1} \in\right] x_{1}, x_{2}[$.

\section{Appendix 3: Numerical Solution to the Test Moving Boundary Problem}

The well-known problem which admits analytic expression for the boundary movement (Ref 4) is given by

$$
\left\{\begin{array}{l}
\frac{\partial u}{\partial t}(x, t)=D \frac{\partial^{2} u}{\partial x^{2}}(x, t) \quad \text { for } 0<x<s(t), \\
s^{\prime}(t)=-\alpha \frac{\partial u}{\partial x}(s(t), t) \quad \text { for } t>0, \\
u(x, 0)=c^{\infty}, \quad u(0, t)=c^{\infty}, \quad u(s(t), t)=c^{\gamma} \\
s(0)=\beta,
\end{array}\right.
$$

where $\alpha=D^{\gamma} /\left(c^{\gamma}-c^{\alpha}\right)$. We can define new variable $v(x, t)=u(s(t) x, t)$ for $0 \leq x \leq 1$. The suitable time and space derivatives of (32) are given as follows: 


$$
\begin{aligned}
& \frac{\partial v}{\partial t}(x, t)=x \frac{\partial u}{\partial x}(s(t) x, t) s^{\prime}(t)+\frac{\partial u}{\partial t}(s(t) x, t) \\
& \frac{\partial v}{\partial x}(x, t)=\frac{\partial u}{\partial x}(s(t) x, t) s(t) \\
& \frac{\partial^{2} v}{\partial x^{2}}(x, t)=\frac{\partial^{2} u}{\partial x^{2}}(s(t) x, t) s^{2}(t) \\
& \frac{\partial v}{\partial t}=x \frac{s^{\prime}}{s} \frac{\partial v}{\partial x}+\frac{D}{s^{2}} \frac{\partial^{2} v}{\partial x^{2}} \\
& \frac{\partial u}{\partial x}(s(t), t) s(t)=\frac{\partial v}{\partial x}(1, t) \\
& s^{\prime}(t) s(t)=-\alpha \frac{\partial v}{\partial x}(1, t) .
\end{aligned}
$$

Using above formulas the problem (32) can be transformed as follows:

$$
\left\{\begin{array}{l}
\frac{\partial v}{\partial t}=\frac{D}{s^{2}(t)} \frac{\partial^{2} v}{\partial x^{2}}+\frac{s^{\prime}(t)}{s(t)} x \frac{\partial v}{\partial x}, \\
s^{\prime}(t)=-\frac{\alpha}{s(t)} \frac{\partial v}{\partial x}(1, t), \quad s(0)=\beta, \\
v(x, 0)=c^{\infty}, \quad v(0, t)=c^{\infty}, \quad v(1, t)=c^{\gamma}, \\
\text { for } x \in[0,1], \quad t \geq 0 .
\end{array}\right.
$$

Defining the auxiliary function $\xi(t)=s(t)^{2}$ and consequently $\xi^{\prime}(t)=2 s(t) s^{\prime}(t)=-2 \alpha(\partial v) /(\partial x)(1, t)$ and introducing them into (34), we obtain:

$$
\left\{\begin{array}{l}
\frac{\partial v}{\partial t}=\frac{D}{\xi(t)} \frac{\partial^{2} v}{\partial x^{2}}+\frac{1}{2} x \frac{\xi^{\prime}(t)}{\xi(t)} \frac{\partial v}{\partial x} \quad \text { for } 0<x<1, \\
\xi^{\prime}(t)=-2 \alpha \frac{\partial v}{\partial x}(1, t), \quad \xi(0)=\beta^{2}, \\
v(x, 0)=c^{\infty}, \quad v(0, t)=c^{\infty}, \quad v(1, t)=c^{\gamma} .
\end{array}\right.
$$

Problem (35) has been solved using (standard methods for partial differential equations) method of lines (Ref 17) and Radau II method for stiff system of ODEs (Ref 19).

\section{References}

1. A.J. Chorin and J.E. Marsden, A Mathematical Introduction to Fluid Mechanics, Springer-Verlag, New York, 2000 (corrected fourth printing)

2. W. Nernst, Die elektromotorische Wirksamkeit der Jonen, Z. Phys. Chem., 1889, 4(2), p 129-181
3. M. Planck, Über die Potentialdifferenz zwischen zwei verdünnten Lösungen binärer Elektrolyte, Ann. Phys. Chem., 1890, 40, p 561-576

4. J.S. Kirkaldy and D.J. Young, Diffusion in the Condensed State, The Institute of Metals, London, 1987

5. R. Filipek, Interdiffusion in Non-Ideal Systems, Arch. Metall. Mater, 2004, 49(2), p 201-218

6. G.E. Murch, Diffusion Kinetics in Solids, Phase Transformations in Materials, G. Kostorz, Ed., Wiley-VCH, Weinheim, 2001, p 171-239

7. M. Danielewski and M. Wakihara, Kinetic Constraints in Diffusion, Defect Diffus.Forum, 2005, 237-240, p 151-156

8. C.D. Levermore, Relating Eddington Factors to Flux Limiters, J. Quant. Spectrosc. Radiat. Transf., 1984, 31, p 149-160

9. M. Zakari and D. Jou, Nonequilibrium Lagrange Multipliers and HeatFlux Saturation, J. Non-Equilib. Thermodyn., 1995, 20, p 342-349

10. E.M. Epperlein, G.J. Rickard, and A.R. Bell, Two-Dimensional Nonlocal Electron Transport in Laser-Produced Plasmas, Phys. Rev. Lett., 1988, 61, p 2453-2456

11. G.J. Rickard, A.R. Bell, and E.M. Epperlein, 2D Fokker-Planck Simulations of Short-Pulse Laser-Plasma Interactions, Phys. Rev. Lett., 1989, 62, p 2687-2690

12. A.M. Anile and S. Pennisi, Thermodynamic Derivation of the Hydrodynamical Model for Charge Transport in Semiconductors, Phys. Rev. B, 1992, 46, p 13186-13193

13. J. Stefan, Über einige Probleme der Theorie der Wärmeleitung, Sitzungsberichte der Mathematisch-Naturwissenschaftliche der Kaiserlichen Adad, Der Wissenschaften, 1889, 98, p 473-484

14. J. Dantzig and M. Rappaz, Solidification (Engineer Sciences: Materials), EFPL Press, Lausanne, 2009

15. W.D. Murray and F. Landis, Numerical and Machine Solutions of Transient Heat Conduction Problems Involving Melting and Freezing, Trans. ASME C J. Heat Transf., 1959, 81, p 106-112

16. L.S. Darken, Diffusion of Carbon in Austenite with a Discontinuity in Composition, Trans. AIME, 1948, 180, p 430-438

17. J.W. Thomas, Numerical Partial Differential Equations-Finite Difference Methods, Springer-Verlag, New York, 1995, p 5-40

18. E. Hairer, S.P. Norsett, and G. Wanner, Solving Ordinary Differential Equations I: Nonstiff Problems, 2nd ed., Springer, Berlin, 2002

19. E. Hairer and G. Wanner, Stiff Differential Equations Solved by Radau Methods, J. Comput. Appl. Math., 1999, 111, p 93-111

20. P. Skrzyniarz, A. Sypień, J. Wojewoda-Budka, R. Filipek, and P. Zięba, Microstructure and Kinetics of Intermetallic Phases Growth in Ag/Sn/ Ag Joint Obtained as the Result of Diffusion Soldering, Arch. Metall. Mater., 2010, 5(5), p 123-130

21. S. Bader, W. Gust, and H. Hieber, Rapid Formation of Intermetallic Compound by Interdiffusion in the $\mathrm{Cu}-\mathrm{Sn}$ and Ni-Sn Systems, Acta Metall. Mater., 1995, 43, p 329-337

22. T.L. Su, L.C. Tsao, S.Y. Chang, and T.H. Chuang, Morphology and Growth Kinetics of Ag3Sn During Soldering Reaction Between Liquid Sn and an Ag Substrate, J. Mater. Eng. Perform., 2002, 11, p 365-368

23. D.R. Flanders, E.G. Jacobs, and R.F. Pinizzotto, Activation Energies of Intermetallic Growth of Sn-Ag Eutectic Solder on Copper Substrates, J. Electron. Mater., 1967, 26, p 883-887 